\title{
Influence of fiducial marker for MR image of prostate radiotherapy
}

Osamu Tanaka $^{1 *}$, Hisao Komeda ${ }^{2}$, Shigeki Hirose ${ }^{3}$, Takayoshi Iida ${ }^{1}$, Eiichi Yama ${ }^{2}$, Hironori Ichihashi RT ${ }^{3}$, Masayoshi Tamaki ${ }^{2}$, Kensaku $^{2}$ Seike $^{2}$ and Shota Fujimoto ${ }^{2}$

${ }^{1}$ Department of Radiation Oncology, Gifu Municipal Hospital, Japan

${ }^{2}$ Department of Urology, Gifu Municipal Hospital, Japan

${ }^{3}$ Division of Radiation Service, Gifu Municipal Hospital, Japan

\begin{abstract}
Fiducial marker for registration to CT and MRI in intensity modulated radiation therapy for prostate cancer came to be increasing. Whereas diffusion weighted image came to be used similar to the PSA of the blood test for radiotherapy effect measurement for prostate cancer. Generally, the diffusion weighted image was often affected by a magnetic component.

We evaluated how much the marker had an influence on the diffusion weighted image. As a result, it was degree in range 2-5 mm that detained a marker that marker affected the image. We concluded that a fiducial marker does not affect the quality of diffusion weighted image on daily practice.
\end{abstract}

Abbreviations: CT: Computed Tomography, MRI: Magnetic Resonance Images, T2-WI: T2 Weighted Image, T2*-WI: T2* Weighted Image, DWI: Diffusion Weighted Image, ADC: Apparent Diffusion Coefficient

\section{Introduction}

The radiotherapy for prostate cancer progressed by onset of the intensity radiotherapy (Intensity modulated radiotherapy; IMRT) drastically. More precise radiation was required with it, and it increased that IMRT was performed as a mark with a gold marker. Also, the reports that a diffusion weighted image [Diffusion weighed image; DWI (apparent diffusion coefficient, ADC level)] of the MRI correlated with Prostate Specific Antigen (PSA) as a radiotherapy effect on prostate cancer increased [1-3].

However, size or the iron component of the marker may influence an image when marker detains you in prostate. DWI in particular was easy to be affected by the metal, and the report that it was precise, and an image was obtained after the custody if an ADC level was before the marker custody Kim or compared was not found until now.

It is thought that an ADC level and an ADC level before the radiotherapy after the treatment are not evaluated fairly when an ADC level of the point in time when marker is not detained changes by the marker custody. In other words, if scenery obtaining DWI before radiotherapy is different, is right; may not evaluate it. We examined the case of 3 patterns to be found clinically primarily this time.

\section{Materials and methods}

We examined the case of 3 patterns as follows

1) The case that the decrease of the ADC level was not seen in from a beginning
2) The case that an ADC level increased to a normal tissue and the degree at the radiotherapy initiation by hormonal therapy before the radiotherapy

3) The case that a picture of DWI of the MRI did not turn into in approximately the custody of marker

We detained VISICOIL ( $0.35 \mathrm{~mm}$ in diameter, $10 \mathrm{~mm}$ in length) which was most often used for this country and Gold Anchor $(0.28$ $\mathrm{mm}$ in diameter, $10 \mathrm{~mm}$ in length) where we made visibility by the MRI by containing iron about marker Kim. We conduct an MRI image-controlled trial of VISICOIL and Gold Anchor and detain Gold Anchor now on VISICOIL, the anti-side in the one side in this hospital. The marker avoided the site where a clear tumor was seen with an image as much as possible to prevent the dissemination of tumor and detained you.

\section{Image acquisition}

Parameters for $\mathbf{T} 2^{\star}$-WI: $\mathrm{T} 2^{\star}$-weighted fast spin-echo [repetition time (TR)/echo time (TE) in ms]: (700/18); number of sample (signals) averaged (NSA): two times; number of phase encoding steps (PES): 205; number of frequency encoding steps (FES): 256; and typical spatial resolution (TPR); frequency/phase: 0.63/0.78.

${ }^{*}$ Correspondence to: Osamu Tanaka, Gifu Municipal Hospital, 7-1 Kashimacho, Gifu city Gifu, 500-8513, Japan, Tel: +81-58-251-1101; Fax: +81-58-2521335 E-mail: c.bluered@gmail.com

Key words: prostate cancer, radiotherapy, MRI, fiducial marker, diffusion weighted image

Received: January 20, 2018; Accepted: March 15, 2018; Published: March 20, 2018 
Parameters for diffusion-WI: Diffusion-weighted gradient-echo [repetition time (TR)/echo time (TE) in ms]: (2264/70); number of sample (signals) averaged (NSA): eight times; number of phase encoding steps (PES): 103; number of frequency encoding steps (FES):128; and typical spatial resolution (TPR); frequency/phase:2.58/3.21.

Parameters for planning CT: Slice thickness $1.25 \mathrm{~mm}$, field of view $40 \mathrm{~cm} \times 40 \mathrm{~cm}, 460 \mathrm{~mA}, 120 \mathrm{kV}$.

Parameters for cone-beam CT: Slice thickness $2.5 \mathrm{~mm}$, Number of pixels $384 \times 384,80 \mathrm{~mA}, 125 \mathrm{kV}$.

CT (Optima CT580, GE Medical Systems, Milwaukee, WI, USA) and MRI (Intera 1.5 Nova, Philips Medical Systems, Eindhoven, The Netherlands) was used.

One blinded radiotherapist conducted the measurement of the ADC. Also, we decided that Region of interest (ROI) compared the image before and after the treatment and surrounded the outline similarly in the site.

1) The case that the decrease of the ADC level was not seen in from a beginning

The patients who visited a hospital for treatment for follow-up for a 60 s man, chronic hepatitis $\mathrm{C}$ in this hospital. PSA rises by an examination with $9.3 \mathrm{ng} / \mathrm{ml}$, and we underwent needle biopsy with 16 , but there is no depiction of cancer. The PSA rises to $13.2 \mathrm{ng} / \mathrm{ml}$ one year later and performs a biopsy. Prostate cancer was detected to one of 16 and was Gleason Score(GS) 5+4 and high level. Also, because there was not the depiction of clear cancer, we took it as T1cN0M0 in the MRI and planned radiotherapy of $78 \mathrm{~Gy} / 39 \mathrm{fx}$. We imaged DWI in the next if before the VISICOIL ( $0.35 \mathrm{~mm}$ in diameter) custody, respectively, but the skewness of the clear image was not found with before and after either (Figure 1).

2) The case that an $\mathrm{ADC}$ level increased at the radiotherapy initiation by hormonal therapy before the radiotherapy

Because a 60s man, dysuria were detected, we receive an urology department. PSA $20.2 \mathrm{ng} / \mathrm{ml}$ and increase. Prostate cancer was detected in a biopsy to three of 16 and had a diagnosis of T3bN0M0 because extracapsular extension was detected in the MRI. We judged GS from 4+4 with high risk, and administration of LH-RH preparationbegan. Indicating the MRI before the administration (Figure $2 \mathrm{a}$ and $2 \mathrm{~b}$ ). One and a half years later, we plan radiotherapy subsequently because decreases of the PSA do not become $4 \mathrm{ng} / \mathrm{ml}$ or less. A tumor lesion disappeared after treatment as compared with an image before the treatment with $\mathrm{LH}-\mathrm{RH}$ preparation. Indicating CT that detained Gold Anchor (0.28 $\mathrm{mm}$ in diameter) and the MRI (Figure 2c-2e). Because we contain iron, the marker is depicted clearly in T2*-WI. The skewness of the image was not found around Gold Anchor in DWI (Figure 2e). The radiotherapy accomplished it in $78 \mathrm{~Gy} / 39 \mathrm{fx}$.

3) The case that the quality of the image did not turn into in approximately the custody of marker

Men in 60s. Because PSA was $13.2 \mathrm{ng} / \mathrm{ml}$ and high level by an examination. We receive this hospital urology department. Prostate cancer was detected to 15 of 16 , and GS was $3+4$ by a biopsy. A right lobe shows the decrease (mean $\mathrm{C}=0.758^{\star} 10-3 \mathrm{~mm}^{2} / \mathrm{sec}$ ) of the ADC level in DWI. We detain VISICOIL subsequently for IMRT. We imaged $\mathrm{CT}$ and MRI on an imaging condition similar to before marker custody for IMRT three weeks later. The ADC level was $0.653^{\star} 10-3 \mathrm{~mm}^{2} / \mathrm{sec}$, and the change was not found in an image (Figure 3 ). The radiotherapy accomplished it in 78Gy/39fx.

\section{Comparison of $\mathrm{CT}$ and $\mathrm{MR}$ image between before marker placement and after.}

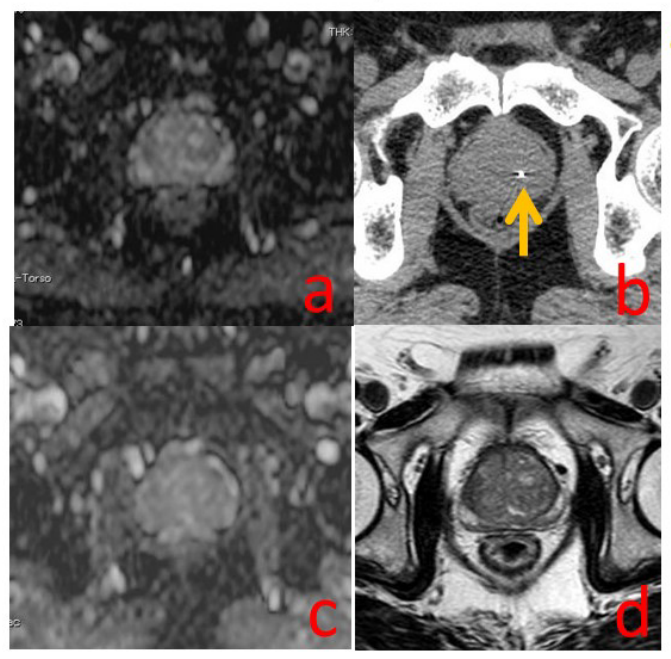

marker

Figure 1. a) DWI of before fiducial marker placement; b) Planning CT of before fiducia marker placement; c) DWI of after fiducial marker placement; d) T2-WI of after fiducial marker placement; e) After placing diameter of $0.35 \mathrm{~mm}$, length $\times 10 \mathrm{~mm}$ marker (VISICOIL), the distortion in DWI of the lesion which detained a marker was not shown.

\section{Comparison of CT and MR image}

between before marker placement and after.

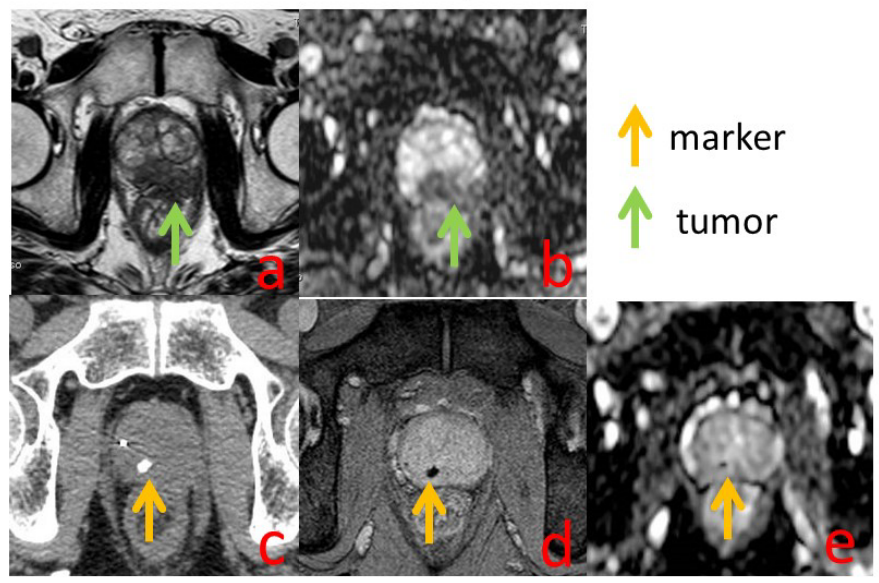

Figure 2. a) T2-WI of before fiducial marker placement; b) DWI of before fiducia marker placement; c) Planning CT of after fiducial marker placement; d) T2*-WI of after fiducial marker placement; e) DWI of after fiducial marker placement

With prostate cancer with the extension extracapsular in the left margins area of the prostate (Figure a,b). The cancer reduced by hormonal therapy and became the size that was not recognizable with the image. We image CT/MRI for the radiation treatment planning for IMRT purpose (after the Gold Anchor custody figure c.d.e). Recognition of Gold Anchor is good in T2*-WI which detained Gold Anchor. Gold Anchor is recognizable, but the neighboring images are not warped in DWI either.

A prostate cancer with the extension extracapsular in the peripheral zone of the prostate (Figure a,b). The cancer reduced by hormonal therapy. A physician makes IMRT planning using CT and MRI fusion (Figure c,d) after the marker placement. The recognition of the marker (Gold Anchor) is clear in T2*-WI which detained a marker. The marker is recognizable in DWI, and the neighboring images are not distorted (Figure e).

\section{Discussion}

With the increase of patients with recent prostate cancer, information came to be able to be obtained in precision about various kinds of modality including the effect of treatment judgment diagnostic method and the regimen of the prostate. Treatment in the present is 


\section{Comparison of CT and MR image between before marker placement and after.}

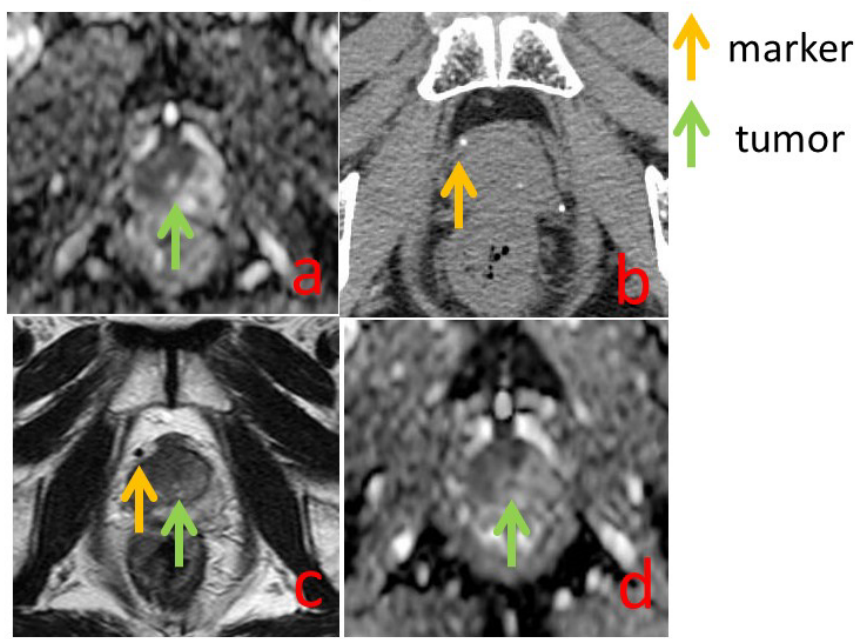

Figure 3: a) WI of before fiducial marker placement; b) Planning CT of after fiducial marker placement; c) T2-WI of after fiducial marker placement; d) DWI of after fiducial marker placement

There is low signal intensity on right transitional zone of the prostate (Figure a). A marker is placed on the capsule of right apex of the prostate (Figure b,c,d). After placement, There were no difference visually between before and after of marker placement (Figure a and d).

endocrine therapy, a surgical remedy, radiotherapy primarily, and a PSA level is used as a Merkmal of the recurrence after progress before the treatment and the treatment. The report that how FDG-PET and MRI reflected for effect of treatment in the diagnosis instrument of these days came to be accomplished [4-6]. When the increase of the ADC level of the site where prostate cancer is present in significantly correlated with a decrease of the PSA according to the report of Yamaguchi et al. in DWI the radiotherapy half a year later, we report it [1].

We are thought to be able to calculate the ADC level with a fixedquantity level theoretically if we always do siege of ROI similarly. However, it is said that an image is often warped when there are a patient movement examining and metal making a magnetic field non-uniform [7-9]. Intramuscular injection of butylscopolamine is provided ten minutes before testing to stop rectal movement during MR imaging in this hospital.

An MRI image is very useful in the depiction of the outline of the prostate and the marker for IMRT. However, an effect of treatment judgment using the MRI and an effect of treatment prediction are not possible when DWI is not evaluated under the influence of metal. Therefore, we evaluated it using two kinds of markers. Real size is recognized by the MRI (T2*-WI) regardless of around $2 \mathrm{~mm}$ as signal void of around $5 \mathrm{~mm}$ by effect of the susceptibility, and Gold Anchor which is a particularly chalybeate marker is very useful in registration with CT. However, the effect of treatment judgment with the image is not possible when effect of this susceptibility affects the ADC level.
There was signal void of $5 \mathrm{~mm}$ in Gold Anchor in T2*-WI (Figure 2). There was not a skewness to the image in the diffusion weighted image visually either, and the decrease of the real ADC level was not actually found, too. However, because we were recognized as signal void, the metal itself was indistinct in DWI in T2*-WI, but was slightly recognizable. However, the recognizable size was similar to $\mathrm{T} 2^{\star}-\mathrm{WI}$, and it was thought that it was not a level to be affected clinically by marker Kim being detained. In other words, we saw the change of the ADC level after the radiotherapy on the basis of the image that marker Kim was detained and combined it with PSA and were thought to be able to evaluate it in DWI.

However, it is an evaluation using the smallest size called a chalybeate marker (Gold Anchor) of $0.28 \mathrm{~mm}$ in diameter and the gold marker $(0.35 \mathrm{~mm})$ of $0.35 \mathrm{~mm}$ this time. This may have an influence on the diffusion weighted image with the large markers such as $0.75 \mathrm{~mm}$. It is necessary to test even a marker different in the size, and manikin studies will be now in future in this hospital.

\section{Conclusion}

The effect to give the MRI (DWI) image of marker Kim served MRI for follow-up small and was thought to be able to use even DWI as an image evaluation. However, as for the size of the marker performed in this clinical trial, it is thought that the study with the big marker of the size is necessary because this is because it is the short marker of the diameter most with chalybeate $0.28 \mathrm{~mm}$ in diameter and normal $0.35 \mathrm{~mm}$.

\section{References}

1. Yamaguchi H, Hori M, Suzuki O, Seo Y, Isohashi F, et al. (2016) Clinical Significance of the Apparent Diffusion Coefficient Ratio in Prostate Cancer Treatment with Intensity-modulated Radiotherapy. Anticancer Res 36: 6551-6556. [Crossref]

2. Qi WX, Zhang Q, Li P, Zhang XM, Zhang GY, et al. (2016) The predictive role of ADC values in prostate cancer patients treated with carbon-ion radiotherapy: initial clinical experience at Shanghai Proton and Heavy Ion Center (SPHIC). J Cancer Res Clin Oncol 142: 1361-1367. [Crossref]

3. Casares-Magaz O, van der Heide UA, Rørvik J, Steenbergen P, Muren LP (2016) A tumour control probability model for radiotherapy of prostate cancer using magnetic resonance imaging-based apparent diffusion coefficient maps. Radiother Oncol 119: 111-116. [Crossref]

4. Iannelli G, Caivano R, Rago L, Simeon V, Lotumolo A, et al. (2016) Diffusionweighted magnetic resonance imaging in patients with prostate cancer treated with radiotherapy. Tumori 102: 71-76. [Crossref]

5. Decker G, Mürtz P, Gieseke J, Träber F, Block W, et al. (2014) Intensity-modulated radiotherapy of the prostate: dynamic ADC monitoring by DWI at 3.0 T. Radiother Oncol 113: 115-120. [Crossref]

6. Morgan VA, Riches SF, Giles S, Dearnaley D, deSouza NM (2012) Diffusion-weighted MRI for locally recurrent prostate cancer after external beam radiotherapy. AJR Am J Roentgenol 198: 596-602. [Crossref]

7. Park SY, Kim CK, Park BK, Park W, Park HC, et al. (2012) Early changes in apparent diffusion coefficient from diffusion-weighted MR imaging during radiotherapy for prostate cancer. Int J Radiat Oncol Biol Phys 83: 749-755. [Crossref]

8. Song I, Kim CK, Park BK, Park W (2010) Assessment of response to radiotherapy for prostate cancer: value of diffusion-weighted MRI at 3 T. AJR Am J Roentgenol 194: W477-W482. [Crossref]

Copyright: (C2018 Tanaka O. This is an open-access article distributed under the terms of the Creative Commons Attribution License, which permits unrestricted use, distribution, and reproduction in any medium, provided the original author and source are credited. 